Alessandra Page Brito ${ }^{1}$ Marina Campos Araujo ${ }^{2}$ Celso Pereira Guimarães ${ }^{3}$ Rosângela Alves Pereira ${ }^{1}$
${ }^{1}$ Departamento de Nutrição Social e Aplicada, Instituto de Nutrição, Universidade Federal do Rio de Janeiro (UFRJ). Av. Carlos Chagas Filho 373/CCS/Bl. J/2,

Cidade Universitária. $21941-$ 590 Rio de Janeiro RJ Brasil.apb.ufrj@gmail.com

${ }^{2}$ Escola Nacional de Saúde Pública Sérgio Arouca, Fiocruz. Rio de Janeiro RJ Brasil

${ }^{3}$ Departamento de Comunicação Visual, UFRJ. Rio de Janeiro RJ Brasil

\section{Validade relativa de questionário de frequência alimentar com suporte de imagens}

\author{
Relative validity of a food frequency questionnaire \\ supported by images
}

Abstract The scope of this study was to evaluate the relative validity of a food frequency questionnaire (FFQ) designed for adolescents. The study involved a sample of 106 adolescents (57\% girls) aged between 11 and 18. The FFQ was administered in one-on-one interviews with the support of the 93 photographs of food items. Overall averages of energy and nutrient intake of three 24hour recalls $(24 h-R)$ were used as benchmarks. They were then compared with those estimated from the FFQ using the Spearman correlation coefficient, weighted kappa for the agreement between classifications into quartiles and Bland-Altman limits of agreement. The correlation coefficients ranged from 0.27 to 0.49 ( mean $=0.43)$, the weighted kappa ranged from 0.28 to 0.51 (mean $=0.40$ ), and the estimated Bland-Altman mean limits of agreement ranged from 109 to 159\% $($ mean $=130 \%)$. Approximately $30 \%$ of the estimates were classified into the same quartile while about $90 \%$ were categorized into the same or adjacent quartiles. Supporting the application of the FFQ with photographs provided more favorable results than those observed for the questionnaire alone. The FFQ was considered appropriate to categorize adolescents according to gradients of dietary intake.

Key words Food consumption, Questionnaire, Photographs, Adolescents, Validity
Resumo O objetivo do presente estudo foi avaliar a validade relativa de questionário de frequência alimentar (QFA), desenvolvido para adolescentes. $O$ estudo envolveu amostra de 106 adolescentes (57\% meninas) de 11 a 18 anos de idade. O QFA foi aplicado em entrevistas pessoais com o suporte de fotografias dos 93 itens alimentares. As médias deatenuadas da ingestão de energia e nutrientes de três recordatórios de 24 horas ( $R 24 h$ ) foram consideradas como referência e foram comparadas com aquelas estimadas pelo QFA com o uso do coeficiente de correlação de Spearman, kappa ponderado para a concordância da categorização em quartis, e limites de concordância de Bland -Altman. Os coeficientes de correlação variaram de 0,27 a 0,49 (média =0,43), o kappa ponderado variou de 0,28 a 0,51 (média =0,40), e a concordância média estimada variou de 109 a 159\% (média $=130 \%$ ). Aproximadamente 30\% das estimativas foram classificados de forma exata em um mesmo quartil e cerca de $90 \%$ em quartis iguais ou adjacentes. Resultados obtidos com a aplicação do QFA com o suporte de fotografias foram mais favoráveis do que os observados para o questionário isoladamente. O QFA foi considerado apropriado para categorizar adolescentes segundo gradientes de consumo energia e nutrientes. Palavras-chave Consumo de alimentos, Questionário, Fotografia, Adolescentes, Validade 


\section{Introdução}

O Questionário de Frequência Alimentar (QFA) permite estimar o consumo alimentar habitual e categorizar os indivíduos de acordo com diferentes gradientes de consumo e, assim, esse instrumento proporciona estimativas das medidas de associação entre os fatores da dieta e a ocorrência de doenças. Além disso, requer apenas uma aplicação e procedimentos relativamente simples para a análise dos dados, o que reduz custos. Por isso, o QFA vem sendo amplamente utilizado em estudos epidemiológicos de larga escala, particularmente estudos de caso-controle e investigações com medidas repetidas, como os estudos de coorte e ensaios clínicos ${ }^{1}$.

O QFA baseia-se em uma lista de alimentos definida de acordo com os hábitos alimentares da população-alvo, portanto, o QFA deve ser desenhado especificamente para a população a que se destina. Porém, na aplicação do QFA são comuns dificuldades na estimativa das quantidades consumidas, sendo essa considerada uma de suas principais limitações, a qual pode comprometer a validade do questionário. Por isso, estudos que avaliam a validade de QFA são altamente recomendados para que, na interpretação de estudos epidemiológicos que empregam o QFA, seja reconhecida a relação entre o consumo relatado e o real ${ }^{2}$.

Para superar as limitações relacionadas com o relato das quantidades consumidas, imagens de alimentos são recomendadas para auxiliar a aplicação do QFA. Fotografias têm a vantagem de que podem ser facilmente adaptadas às circunstâncias locais, além de serem de baixo custo e facilmente reproduzidas e transportadas ${ }^{3,4}$.

No Brasil, já existem alguns álbuns fotográficos elaborados com o intuito de dar suporte aos inquéritos alimentares ${ }^{5-9}$. Porém, são escassos os estudos desenvolvidos no Brasil que analisam a validade de QFA associado a fotografias de alimentos $^{10-13}$.

No país, já existem alguns QFA validados para adolescentes ${ }^{10,12,14-18}$, sendo que um foi desenvolvido no Rio de Janeiro ${ }^{19}$, o qual foi avaliado quanto a sua reprodutibilidade ${ }^{20}$ e validade $^{21}$, tendo apresentado confiabilidade e validade relativa aceitáveis.

Com o objetivo de favorecer o relato das quantidades consumidas na aplicação desse QFA elaborado para adolescentes do Rio de Janeiro ${ }^{19}$, foi elaborado um álbum com as fotografias de todos os itens incluídos na lista de alimentos do QFA, observando técnicas apropriadas para ima- gens de alimentos e as recomendações de Nelson e Haraldsdóttir ${ }^{22}$. As fotografias foram submetidas a estudo de sua validade, no qual verificou-se a validade de mensuração (aspecto inserido na validade de informação $)^{23}$ a fim de observar se os resultados não decorriam de erros sistemáticos obtidos com a utilização do manual fotográfico, sendo analisado então a capacidade dos adolescentes reconhecerem os alimentos e suas porções nas fotografias. Este estudo apresentou resultados satisfatórios, pois $80 \%$ dos alimentos foram identificados corretamente pela totalidade dos adolescentes, $83 \%$ das porções naturais e típicas foram identificadas corretamente por todos os participantes, e $89 \%$ das porções de alimentos amorfos foram identificadas corretamente por pelo menos $60 \%$ dos participantes ${ }^{24}$.

Dessa forma, este estudo teve como objetivo avaliar se a adoção de fotografias como recurso visual para auxiliar o relato das porções consumidas poderia aprimorar a qualidade das estimativas de consumo dietético obtidas com o QFA elaborado para adolescentes do Rio de Janeiro. Com isso, analisou-se a validade relativa do QFA acoplado ao manual fotográfico a fim de verificar sua capacidade de mensuração.

\section{Métodos}

\section{Desenho e população do estudo}

Foram sorteados 120 adolescentes de 11 a 18 anos de idade, do sexto ano do ensino fundamental até o terceiro ano do ensino médio de uma escola municipal de Niterói, Rio de Janeiro, a qual foi selecionada por conveniência. Os adolescentes foram selecionados de modo que houvesse equilíbrio na proporção de adolescentes segundo o sexo ( $50 \%$ meninas) e a faixa de idade (50\% de 11 a 14 anos). Destes, 106 adolescentes apresentaram termos de consentimento livre e esclarecido assinados pelo responsável e participaram de todas as etapas do estudo.

A coleta de dados ocorreu durante o período letivo de 2009. O QFA foi aplicado por meio de entrevista pessoal previamente aos três recordatórios de 24 horas (R24h) não consecutivos, sendo um relativo a um dia de final de semana, com intervalo de três semanas a um mês entre cada R24h.

\section{Questionário de Frequência Alimentar}

Como descrito em publicação anterior ${ }^{19}$, a lista de alimentos do QFA foi elaborada a partir 
de dados de registros alimentares obtidos em estudo de base escolar desenvolvido em 2003 com adolescentes da área da região metropolitana do Rio de Janeiro. Foram incluídos inicialmente 90 itens citados pelo menos 15 vezes nos registros alimentares e que respondiam por $95 \%$ da ingestão total de energia e nutrientes estimada. As porções de referência foram as mais frequentemente referidas no estudo de base. Após o estudo de reprodutibilidade ${ }^{20}$ e validação ${ }^{21}$, foram excluídos os itens que não foram citados e incluídos alimentos referidos com frequência elevada pelos adolescentes no método de referência. Por fim, 93 itens compuseram a lista do QFA.

$O$ QFA foi definido no formato vertical e as opções de frequência foram organizadas de forma diferenciada para os diversos itens do questionário de acordo com a frequência de consumo no estudo de base e no de validade e reprodutibilidade. Para o relato da frequência de consumo de manteiga/margarina, feijão e arroz, que são os mais frequentemente consumidos, as opções variaram de "menos de uma vez por mês" a "seis ou mais vezes ao dia”. Para 15 itens, as opções para o relato da frequência de consumo das porções de referência variaram de "menos de uma vez por mês" a "quatro ou mais vezes ao dia" (por exemplo, leite, café, chá, achocolatado, pão, entre outros). Para 24 itens, foram disponibilizadas opções para relatar o consumo de alimentos variando entre "menos de uma vez por mês" e "duas ou mais vezes ao dia" (exemplo, macarrão, carne e ovo) e para os demais 51 itens, foram disponibilizadas opções para relatar o consumo de alimentos variando entre "menos de uma vez por mês" e "cinco ou mais vezes por semana".

O período de referência foi alterado após o estudo de reprodutibilidade e validade, passando a ser referente aos três meses precedentes à entrevista. A revisão do QFA também considerou informações obtidas em grupos focais desenvolvidos com o objetivo de aprimorar ou facilitar sua aplicação. Os resultados dos grupos focais mostraram, por exemplo, que a interpretação de "semana" não inclui o "final de semana", atribuindo à semana significado relacionado com as atividades escolares e trabalho. $\mathrm{Na}$ aplicação do QFA, os adolescentes eram perguntados sobre a frequência de consumo dos alimentos em todos os dias da semana. As observações dos grupos focais levaram à reformulação da forma de aplicar o questionário tendo em vista reduzir as fontes de erro de compreensão.

\section{Álbum fotográfico}

As fotografias dos alimentos incluídos no QFA foram desenvolvidas pelo Laboratório Núcleo Comunicação Design, da Universidade Federal do Rio de Janeiro - UFRJ. Essa etapa contou com a participação de nutricionistas, que orientaram quanto à aquisição, preparo e porcionamento dos itens fotografados. Cada alimento listado no QFA foi fotografado na sua porção de referência. Quando um item do QFA contemplava mais de um alimento (por exemplo, "biscoito recheado ou waffer" ou "alface ou agrião”), foram fotografados todos os itens questionados. Assim, o álbum fotográfico totalizou 101 fotografias de alimentos.

Na elaboração das fotografias de alimentos, utilizou-se câmara Canon Digital EOS Rebel XT com lentes de EFS 18-55 mm montada sobre tripé. Foram utilizados alimentos e preparações frescos, fotografados sempre a partir de um mesmo ângulo e distância, e foram incluídas medidas de referência escalar (garfo e faca de jantar). Procedimentos realizados no estúdio fotográfico incluíram a adoção de fundo neutro infinito em relação aos diversos produtos, iluminação promovendo contrastes suaves, definição focal e correção da perspectiva (Figura 1).

\section{Método de referência: recordatório de 24 horas}

Os recordatórios de 24 horas foram aplicados considerando uma adaptação do método de múltiplas passagens proposto pelo Departamento de Agricultura dos Estados Unidos da América (USDA) ${ }^{25}$, utilizando fotografias de utensílios e vasilhames com o objetivo de auxiliar o relato das porções consumidas.

Resumidamente, a aplicação do método foi desenvolvida nas seguintes etapas: (a) solicitava-se ao participante uma listagem de todos os alimentos e bebidas consumidos no dia anterior; (b) em seguida, perguntava-se sobre o consumo de alimentos que são usualmente omitidos em recordatórios de 24 horas (açúcar, manteiga/margarina, óleo/azeite, bebidas, pequenos lanches, balas etc.); (c) num terceiro momento, o entrevistado detalhava o horário, o local e a ocasião/refeição em que cada alimento foi consumido; (d) a seguir, o entrevistado era inquirido sobre detalhes dos alimentos relatados, se era regular, diet ou light, a forma de preparação e as quantidades consumidas; (e) a última etapa era constituída pela revisão final das informações e 

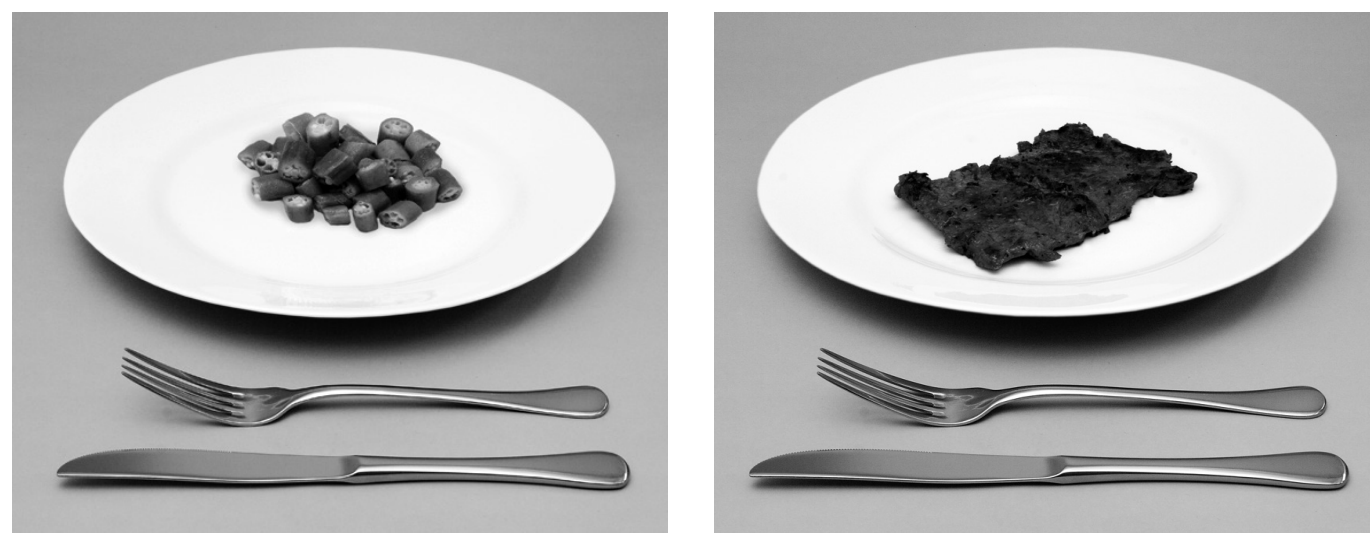

Figura 1. Exemplos de fotografias de alimentos desenvolvidas para suporte de Questionário de Frequência Alimentar desenvolvido para adolescentes do Rio de Janeiro.

nova sondagem sobre alimentos que são frequentemente omitidos em inquéritos alimentares ${ }^{24}$.

\section{Tratamento dos dados e análises estatísticas}

Para a estimativa da ingestão de energia e nutrientes foi utilizado o Programa de Apoio à Nutrição - NutWin (Departamento de Informática em Saúde, Universidade Federal de São Paulo - UNIFESP/Brasil) que se baseia na tabela de composição de alimentos do Departamento de Agricultura dos Estados Unidos da América (USDA http://ndb.nal.usda.gov/), complementando com as informações da Tabela Brasileira de Composição de Alimentos - TACO (http:// www.unicamp.br/nepa/taco/contar/taco_4_edicao_ampliada_e_revisada).

Para a análise do QFA, a frequência de consumo relatada para cada item foi transformada em frequência diária. Para tanto, considerou-se a opção de frequência "uma vez ao dia” como frequência diária igual a '1' (um). As demais opções foram relacionadas proporcionalmente à unidade: por exemplo, para os itens consumidos duas a três vezes ao dia, a frequência diária foi 2,5 [ $2+$ 3)/2] e para itens consumidos duas a quatro vezes por semana, considerou-se frequência semanal média igual a três, que dividido por sete proporcionava a estimativa do consumo médio diário = 0,43. A frequência diária foi usada para estimar a quantidade média (em gramas) consumida por dia para cada alimento (frequência diária x porção de referência), a qual foi utilizada para calcular a ingestão diária de energia e nutrientes.
Os dados obtidos pelo R24h foram corrigidos pela variabilidade intraindividual pelo método da Iowa State University ${ }^{26}$, o qual prevê a estimativa das variâncias intraindividual e interindividual, e a utilização da razão dessas variâncias para deatenuação das estimativas da ingestão de energia e nutrientes. O programa PC-SIDE (Department of Statistics, Iowa State University, Iowa, Estados Unidos) foi utilizado para desenvolver a correção pela variabilidade intraindividual.

Todas as variáveis foram testadas quanto às propriedades de suas distribuições com uso do teste de Kolgomorov-Smirnov e como as variáveis apresentaram distribuições assimétricas, foram estimados os coeficientes de correlação de Spearman entre as estimativas da ingestão de energia e nutrientes segundo o QFA e a média dos três dias de R24h.

As médias e as diferenças entre as estimativas da ingestão de energia e nutrientes obtidas pelo QFA e R24h foram estimadas para os dados transformados em logaritmos para a aplicação do método Bland-Altman ${ }^{27}$ na avaliação da concordância entre duas medidas. Dessa forma, foram calculados os limites de concordância (LOA), que foram determinados por: média das diferenças \pm 1.96 * desvio-padrão da distribuição das diferenças entre os métodos. Para facilitar a interpretação de seus resultados, foram calculados os exponenciais da média das diferenças e dos limites de concordância, resultando em razões que expressam o grau de concordância entre os métodos em percentual. A diferença média representa a concordância média e os limites de 
concordância são os valores que incluem 95\% das diferenças entre os métodos.

Para verificar se a concordância entre os métodos variou com a magnitude do consumo, desenvolveu-se procedimento de regressão linear, tendo como variável dependente a diferença entre as estimativas do QFA e R24h e variável independente a média dos dois métodos. Os coeficientes de regressão demonstram se o viés observado nas estimativas do QFA, seja pela subestimativa ou superestimativa do consumo quando comparado ao método de referência, variam segundo a magnitude do consumo. Ou seja, coeficientes de regressão diferentes de zero e estatisticamente significativos indicam que a concordância varia com a magnitude do consumo ${ }^{28}$.

A ingestão de energia e nutrientes estimada pelo QFA e pela média deatenuada dos três dias de R24h foi categorizada em quartis e estimouse a concordância desta categorização com o uso da estatística kappa ponderado. Adicionalmente, avaliou-se a proporção de adolescentes categorizados no mesmo quartil de consumo entre os dois métodos (concordância exata); os categorizados nos quartis adjacentes (concordância adjacente) e os adolescentes categorizados em quartis distantes e opostos (discordância). Para a avaliação da significância estatística foi considerado o valor de $\mathrm{p}<0,05$.

\section{Aspectos éticos}

A pesquisa foi aprovada pelo Comitê de Ética em Pesquisa do Instituto de Medicina Social da Universidade Estadual do Rio de Janeiro em $13 / 01 / 05$. A participação na pesquisa foi condicionada à assinatura do Termo de Consentimento Livre e Esclarecido por responsável ou pelo indivíduo com idade igual ou maior de 18 anos.

\section{Resultados}

A idade média dos 106 adolescentes investigados (88\% da amostra original) foi 14,5 anos (desvio-padrão $=1,75$ anos), sendo que $56 \%$ desses adolescentes tinham entre 11 a 14 anos de idade, $57 \%(n=60)$ eram meninas e $24 \%$ apresentavam excesso de peso. Não houve diferença estatisticamente significativa na distribuição etária segundo o sexo (dados não mostrados).

Houve redução dos desvios-padrão para as estimativas deatenuadas para energia e todos os nutrientes analisados. Verificou-se que as médias brutas e deatenuadas dos R24h para a ingestão de energia e todos os nutrientes analisados foram menores do que as médias obtidas com o QFA (Tabela 1).

Os coeficientes de correlação de Spearman variaram de 0,25 (colesterol) a 0,48 (energia,

Tabela 1. Médias e desvios-padrão (DP) do consumo de energia e nutrientes estimados para questionário de frequência alimentar com suporte de imagens (QFA) e para estimativas brutas e deatenuadas ${ }^{1}$ obtidas em três recordatórios de 24 horas (R24h). Adolescentes de Niterói-RJ ( $\mathrm{n}=106), 2009$.

\begin{tabular}{|c|c|c|c|c|c|c|}
\hline \multirow{3}{*}{ Energia /nutrientes } & \multirow{2}{*}{\multicolumn{2}{|c|}{ QFA }} & \multicolumn{4}{|c|}{ R24h } \\
\hline & & & \multicolumn{2}{|c|}{ Estimativas brutas } & \multicolumn{2}{|c|}{ Estimativas deatenuadas } \\
\hline & Média & DP & Média & DP & Média & DP \\
\hline Energia (kcal) & 3225 & 1240 & 2428 & 791 & 2437 & 647 \\
\hline Proteína (g) & 101 & 37 & 87 & 29 & 84 & 21 \\
\hline Lipídio (g) & 102 & 44 & 76 & 30 & 76 & 22 \\
\hline Glicídio (g) & 485 & 189 & 348 & 114 & 355 & 98 \\
\hline Colesterol (mg) & 262 & 126 & 215 & 94 & 221 & 55 \\
\hline Cálcio (mg) & 887 & 457 & 566 & 294 & 532 & 207 \\
\hline Ferro (mg) & 16,6 & 6,7 & 13,0 & 4,8 & 13,3 & 3,6 \\
\hline Zinco (mg) & 10,5 & 3,6 & 9,1 & 3,2 & 8,6 & 2,0 \\
\hline Fósforo (mg) & 1330 & 520 & 1012 & 366 & 971 & 265 \\
\hline Folato (mg) & 313 & 128 & 213 & 96 & 218 & 77 \\
\hline Fibra (g) & 22,3 & 8,0 & 13,2 & 5,8 & 13,8 & 4,4 \\
\hline
\end{tabular}

* Correção pela variabilidade intraindividual. 
fósforo e folato) para os dados brutos e de 0,27 (colesterol) a 0,49 (cálcio e fósforo) para as estimativas do R24h corrigidas pela variabilidade intraindividual (dados deatenuados), e em ambos os casos, todos os coeficientes estimados foram estatisticamente significativos (Tabela 2).
As análises segundo o método proposto por Bland-Altman permitiram observar que a concordância média para as estimativas brutas da ingestão de energia e nutrientes variou de 114\% (proteína) a 173\% (fibra) e, para as estimativas deatenuadas pela variabilidade intraindividual,

Tabela 2. Coeficientes de correlação de Spearman (r), concordância média, limites de concordânciaa e coeficiente de regressão obtidos na comparação entre estimativas brutas e deatenuadas obtidas com questionário de frequência alimentar com suporte de imagens e a média de três recordatórios de 24-horas. Adolescentes de Niterói-RJ ( $\mathrm{n}=106), 2009$.

\begin{tabular}{|c|c|c|c|c|}
\hline $\begin{array}{c}\text { Energia / } \\
\text { Nutrientes }\end{array}$ & $\mathbf{r}^{\mathrm{b}}$ & $\begin{array}{l}\text { Concordância } \\
\text { média }(\%)^{c}\end{array}$ & $\begin{array}{c}\text { Limites de } \\
\text { concordância }(\%)^{\mathrm{d}}\end{array}$ & $\begin{array}{c}\text { Coeficiente de } \\
\text { regressão }^{\mathrm{e}} \\
\boldsymbol{\beta}\end{array}$ \\
\hline \multicolumn{5}{|l|}{ Estimativas brutas } \\
\hline Energia (kcal) & 0,48 & 130 & $62 ; 272$ & 0,21 \\
\hline Proteína (g) & 0,44 & 114 & $54 ; 243$ & 0,15 \\
\hline Lipídio (g) & 0,42 & 133 & $54 ; 327$ & 0,02 \\
\hline Glicídio (g) & 0,47 & 136 & $65 ; 287$ & $0,25^{*}$ \\
\hline Colesterol (mg) & 0,25 & 120 & $38 ; 376$ & 0,14 \\
\hline Cálcio (mg) & 0,47 & 160 & $50 ; 519$ & $-0,15$ \\
\hline Ferro (mg) & 0,42 & 125 & $55 ; 285$ & 0,15 \\
\hline Zinco (mg) & 0,40 & 117 & $53 ; 255$ & $-0,09$ \\
\hline Fósforo (mg) & 0,48 & 130 & $59 ; 287$ & 0,05 \\
\hline Folato (mg) & 0,48 & 149 & $59 ; 375$ & $-0,04$ \\
\hline Fibra (g) & 0,40 & 173 & $72 ; 414$ & $-0,13$ \\
\hline Média & 0,43 & 135 & & \\
\hline \multicolumn{5}{|c|}{ Estimativas deatenuadas ${ }^{\mathrm{f}}$} \\
\hline Energia (kcal) & 0,48 & 127 & $63 ; 256$ & $0,48^{* *}$ \\
\hline Proteína (g) & 0,44 & 116 & $58 ; 232$ & $0,54^{* *}$ \\
\hline Lipídio (g) & 0,44 & 128 & $58 ; 281$ & $0,47^{* *}$ \\
\hline Glicídio (g) & 0,45 & 131 & $64 ; 271$ & $0,49^{* *}$ \\
\hline Colesterol (mg) & 0,27 & 109 & $41 ; 290$ & $0,96^{* *}$ \\
\hline Cálcio (mg) & 0,49 & 159 & $58 ; 434$ & $0,31^{*}$ \\
\hline Ferro (mg) & 0,44 & 119 & $57 ; 251$ & $0,53^{* *}$ \\
\hline Zinco (mg) & 0,41 & 118 & $61 ; 229$ & $0,52^{* *}$ \\
\hline Fósforo (mg) & 0,49 & 132 & $64 ; 270$ & $0,44^{* *}$ \\
\hline Folato (mg) & 0,47 & 138 & $60 ; 320$ & $0,35^{* *}$ \\
\hline Fibra (g) & 0,38 & 157 & $73 ; 342$ & $0,33^{*}$ \\
\hline Média & 0,43 & 130 & & \\
\hline
\end{tabular}

a Os valores apresentados são os exponenciais da concordância média e dos limites de concordância, visto que os dados foram transformados em logaritmos para a obtenção dessas estimativas. ${ }^{\mathrm{b}} \mathrm{r}=$ coeficiente de correlação de Spearman ( $\mathrm{p}<0.05$ para todas as estimativas). ${ }^{\mathrm{c}}$ Concordância média $=$ média das diferenças entre as estimativas obtidas com o questionário de frequência alimentar e com os recordatórios de 24 horas. ${ }^{\circledR}$ Limites de concordância $=$ média das diferenças $\pm 1,96 \mathrm{x}$ desvio-padrão da distribuição das diferenças. ${ }^{\mathrm{e}}$ Coeficientes obtidos em análise de regressão linear entre as médias (variável independente) e as diferenças (variável dependente) das estimativas obtidas com o questionário de frequência de consumo e os recordatórios de 24 horas. ${ }^{\mathrm{f}}$ Correção pela variabilidade intraindividual. . ${ }^{*} \mathrm{p}<0,05 ;{ }^{* *} \mathrm{p}<0,01$. 
a concordância média variou de 109\% (colesterol) a $159 \%$ (cálcio), com média de $130 \%$. Os coeficientes de regressão entre as diferenças e as médias da ingestão de energia e nutrientes demonstraram o grau do viés do QFA quando comparado ao método de referência. Verificou-se que os coeficientes foram estatisticamente significativos para glicídio bruto e para energia e todos os nutrientes deatenuados, demonstrando que a superestimativa do QFA na ingestão dos nutrientes foi dependente da magnitude do consumo, sendo maior conforme o aumento da ingestão dos nutrientes (Tabela 2). A Figura 2 exemplifica a representação gráfica da análise de Bland-Altman para a ingestão de energia deatenuada. Nesse exemplo, observa-se que o QFA superestimou a ingestão de energia quando comparado à média dos R24h e que esse viés da superestimativa foi mais evidente para valores mais elevados de ingestão de energia.

O kappa ponderado para a concordância das estimativas brutas da ingestão de energia e nutrientes variou de 0,28 (colesterol) a 0,47 (fósforo) e para os dados deatenuados pela variabilidade intraindividual, de 0,28 (colesterol) a 0,51 (cálcio); os intervalos de confiança de 95\% para os kappas mostraram que todos são diferentes de zero, sendo que os menores limites inferiores foram para colesterol, zinco e fibra (Tabela 3 ).

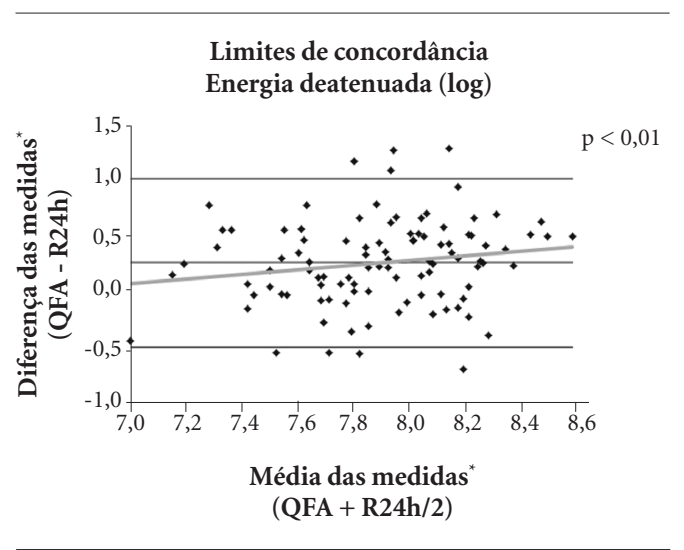

Figura 2. Concordância média e limites de concordância superior e inferior entre a ingestão de energia (kcal) deatenuado estimada por meio de questionário de frequência alimentar com suporte de imagens (QFA) e pela média de três recordatórios de 24-horas. Adolescentes de Niterói-RJ ( $\mathrm{n}=106), 2009$.

* dados transformados em logaritmos.
A concordância exata na categorização em quartis para as estimativas brutas variou de $23 \%$ (zinco) a 33\% (proteína) e de 23\% (colesterol) a $31 \%$ (proteína e glicídio) para as estimativas deatenuadas. A categorização em um mesmo quartil e em quartis adjacentes variou de $83 \%$ (colesterol) a $94 \%$ (fósforo) para as estimativas brutas e de $81 \%$ (colesterol) a 93\% (fósforo) para as estimativas deatenuadas. A discordância na categorização em quartis (quartis opostos) variou entre $6 \%$ (fósforo) e 17\% (colesterol) para as estimativas brutas e entre 7\% (fósforo) e 19\% (colesterol) para as estimativas corrigidas pela variabilidade intraindividual (Tabela 3).

\section{Discussão}

O presente estudo verificou média de coeficientes de correlação de Spearman entre as estimativas da ingestão de energia e nutrientes obtidas com o QFA (com suporte de imagem) e três R24h igual a 0,43 , tanto para as estimativas brutas como para as deatenuadas pela variabilidade intraindividual. E a concordância média entre os dois instrumentos estimada pelo método de Bland-Altman ${ }^{27}$ foi de $130 \%$, o que demonstra que em média o QFA superestimou a ingestão dos nutrientes analisados quando comparado ao método de referência e essa superestimativa aumentou de acordo com o incremento do consumo dos nutrientes. O Kappa ponderado estimado para avaliar a concordância da classificação em quartis segundo ambos os métodos teve média de 0,41 , sendo que aproximadamente 30\% das estimativas foram classificadas de forma exata em um mesmo quartil e cerca de $90 \%$ em quartis iguais ou adjacentes.

Segundo a classificação do Kappa proposta por Landis e Koch ${ }^{29}$, as concordâncias observadas foram moderadas para energia, proteína, lipídio, glicídio, cálcio, ferro, fósforo e folato (kappa entre 0,41 a 0,60 ) e regular para a ingestão de colesterol, zinco e fibra (kappa entre 0,21 a 0,40), não sendo verificada concordância fraca para nenhum nutriente.

Nesta análise, foram desenvolvidos procedimentos comparáveis aos empregados na avaliação da validade da versão original desse QFA, aplicada sem o suporte de fotografias de alimentos, na qual se adotou como método de referência a média de três dias de registros alimentares. Os coeficientes de correlação foram inferiores aos observados neste estudo para energia $(0,41$ vs. 0,48$)$, proteína $(0,34$ vs. 0,44$)$, lipídio $(0,33$ vs. $0,42)$, colesterol $(0,19$ vs. 0,25$)$, cálcio $(0,40$ vs. 
Tabela 3. Kappa ponderado (intervalo de confiança - IC 95\%) e concordância da categorização em quartis das estimativas brutas e deatenuadas ${ }^{1}$ do consumo de energia e nutrientes obtidas por questionário de frequência alimentar com suporte de imagens e a média de três recordatórios de 24 horas. Adolescentes de Niterói-RJ $(\mathrm{n}=106), 2009$.

\begin{tabular}{|c|c|c|c|c|c|}
\hline Energia/Nutrientes & Kappa ponderado & IC 95\% & $\begin{array}{c}\text { Concordância } \\
\text { exata }^{\mathrm{b}} \\
(\%)\end{array}$ & $\begin{array}{c}\text { Concordância } \\
{\text { exata }+ \text { adjacente }^{\mathrm{c}}}_{(\%)}\end{array}$ & $\begin{array}{c}\text { Discordância }^{\mathrm{d}} \\
(\%)\end{array}$ \\
\hline \multicolumn{6}{|l|}{ Estimativas brutas } \\
\hline Energia (kcal) & 0,40 & 0,$23 ; 0,57$ & 30 & 89 & 10 \\
\hline Proteína (g) & 0,42 & 0,$24 ; 0,60$ & 33 & 92 & 9 \\
\hline Lipídio (g) & 0,45 & 0,$29 ; 0,61$ & 29 & 91 & 9 \\
\hline Glicídio (g) & 0,44 & 0,$28 ; 0,60$ & 30 & 88 & 12 \\
\hline Colesterol (mg) & 0,28 & 0,$11 ; 0,45$ & 24 & 83 & 17 \\
\hline Cálcio (mg) & 0,46 & 0,$31 ; 0,61$ & 26 & 89 & 12 \\
\hline Ferro (mg) & 0,40 & 0,$24 ; 0,56$ & 26 & 89 & 12 \\
\hline Zinco (mg) & 0,32 & 0,$15 ; 0,49$ & 23 & 89 & 12 \\
\hline Fósforo (mg) & 0,47 & 0,$31 ; 0,63$ & 30 & 94 & 6 \\
\hline Folato (mg) & 0,45 & 0,$29 ; 0,61$ & 30 & 90 & 10 \\
\hline Fibra (g) & 0,34 & 0,$17 ; 0,51$ & 26 & 89 & 11 \\
\hline Média & 0,40 & & 28 & 89 & 11 \\
\hline \multicolumn{6}{|c|}{ Estimativas deatenuadas ${ }^{\mathrm{a}}$} \\
\hline Energia (kcal) & 0,42 & 0,$25 ; 0,59$ & 29 & 89 & 11 \\
\hline Proteína (g) & 0,41 & 0,$21 ; 0,57$ & 31 & 90 & 10 \\
\hline Lipídio (g) & 0,46 & 0,$30 ; 0,62$ & 28 & 91 & 9 \\
\hline Glicídio (g) & 0,41 & 0,$22 ; 0,58$ & 31 & 89 & 11 \\
\hline Colesterol (mg) & 0,28 & 0,$11 ; 0,45$ & 23 & 81 & 19 \\
\hline Cálcio (mg) & 0,51 & 0,$37 ; 0,65$ & 26 & 89 & 11 \\
\hline Ferro (mg) & 0,41 & 0,$25 ; 0,57$ & 27 & 86 & 14 \\
\hline Zinco (mg) & 0,31 & 0,$14 ; 0,48$ & 24 & 89 & 11 \\
\hline Fósforo (mg) & 0,50 & 0,$34 ; 0,66$ & 30 & 93 & 7 \\
\hline Folato (mg) & 0,44 & 0,$27 ; 0,61$ & 30 & 90 & 10 \\
\hline Fibra (g) & 0,32 & 0,$14 ; 0,50$ & 26 & 87 & 13 \\
\hline Média & 0,41 & & 28 & 89 & 11 \\
\hline
\end{tabular}

${ }^{a}$ Correção pela variabilidade intraindividual. ${ }^{\text {b }}$ Classificação em um mesmo quartil. c Classificação em um mesmo quartil e em quartis adjacentes. ${ }^{\mathrm{d}}$ Classificação em quartis opostos.

0,47), ferro (0,34 vs. 0,42$)$, zinco $(0,28$ vs. 0,40$)$, fósforo $(0,36 v s .0,48)$ e folato $(0,45$ vs. 0,48$)$, e similares para glicídio $(0,46$ vs. 0,47$)$ e fibra $(0,41$ vs. 0,40$)^{21}$.

Araujo et al. ${ }^{21}$ observaram concordâncias médias de Bland-Altman que indicavam que o QFA superestimava a ingestão de energia e nutrientes em $44 \%$, enquanto que com o QFA com suporte de imagens a superestimativa da ingestão de energia e nutrientes foi, em média, de $30 \%$. Além disso, a classificação das ingestões de energia e nutrientes estimadas pelo QFA e pelo R24h em quartis opostos foi, em média, 2,5 vezes maior quando o QFA foi aplicado sem o apoio de fotografias (média de 28\% para o QFA original versus média de $12 \%$ no presente estudo). Estes achados mostram que a utilização de fotografias efetivamente proporcionou melhor estimativa do consumo dietético.

Os resultados deste estudo são comparáveis aos dois que investigaram a validade de QFA aplicado com suporte de fotografias entre adolescentes brasileiros, sendo um desenvolvido na Bahia ${ }^{10}$ e outro em Porto Alegre ${ }^{12}$.

Matos et al. ${ }^{10}$ investigaram a validade de QFA com 98 alimentos entre 108 crianças e adolescentes de 4 a 11 anos de idade da Bahia. Álbum com desenhos de alimentos e utensílios foi utilizado para minimizar erros na estimativa da quantidade consumida. No geral, esses autores 
observaram coeficientes de correlação (média = 0,29 ) e concordância Kappa (média $=0,23$ ) inferiores aos estimados no presente estudo. Henn et al. ${ }^{12}$ avaliaram a validade de QFA com 135 itens e aplicado juntamente com álbum fotográfico entre adolescentes $(n=125)$, adultos $(n=66)$ e idosos $(\mathrm{n}=47)$ de Porto Alegre e obtiveram estimadores semelhantes aos observadas neste estudo, por exemplo, a média dos coeficientes de correlação estimados para os dados deatenuados de ingestão de energia e nutrientes foi 0,44 . No entanto, observaram proporção média de indivíduos classificados em um mesmo quartil ou quartis adjacentes inferiores às verificadas neste estudo para os dados brutos (74,6\% vs. 89,4\%).

Outros estudos que validaram o QFA com manual fotográfico no Brasil ${ }^{11,13}$ não privilegiaram análises com adolescentes. Zanolla et al. ${ }^{11}$ avaliaram a validade do QFA em adultos de Porto Alegre, no qual os participantes dimensionavam o tamanho da porção usualmente consumida com a ajuda de um álbum fotográfico. Os autores observaram resultados comparáveis ao presente estudo, sendo a média das estatísticas Kappa igual a 0,25 e cerca de $36 \%$ das estimativas foram classificadas em um mesmo quartil. Crispim et al. ${ }^{13}$ analisaram a validade de QFA com manual fotográfico desenvolvido para adultos da cidade de Viçosa, Minas Gerais, e obtiveram média dos coeficientes de correlação igual a 0,52 para os dados deatenuados.

Os resultados obtidos também são semelhantes aos observados em estudos internacionais que avaliaram QFA com suporte de fotografias desenvolvidos para adolescentes. Em estudo com 170 adolescentes da Malásia, Nurul-Fadhilah et al. ${ }^{30}$ verificaram a validade e a reprodutibilidade de QFA com 124 itens acoplado a manual fotográfico. Os autores observaram resultados comparáveis aos obtidos no presente estudo, destacando que o QFA também superestimou a ingestão de energia e nutrientes quando comparado à média de três R24h. Similarmente, a mediana dos coeficientes de correlação calculados entre as estimativas dos dois métodos foi de 0,43 ; entretanto a proporção mediana da classificação cruzada em um mesmo quartil ou quartis adjacentes foi de $52,7 \%$, menor do que a estimada neste estudo.

Kobayashi et al. ${ }^{31}$, em estudo realizado com crianças e adolescentes japoneses de 3 a 16 anos de idade $(\mathrm{n}=89)$, avaliaram a validade de QFA de 75 itens aplicado com suporte de imagens para cada alimento do questionário e concluíram que seria necessário desenvolver um novo questionário ou modificar o instrumento avaliado.
Em estudo desenvolvido com 168 meninas chinesas entre 12 e 18 anos, Xia et al. ${ }^{32}$ avaliaram QFA de 86 itens aplicado com suporte de fotografias comparado com a média de nove conjuntos de três recordatórios de 24-horas. Na classificação cruzada em quartis, os autores verificaram concordância moderada (Kappa variou entre 0,4 a 0,6, com média $=0,42$ ) para quase todos os nutrientes e alimentos analisados e estes achados foram similares ao observado no presente estudo. Entretanto, em geral, foram estimados coeficientes de correlação maiores do que os obtidos no presente estudo, os quais variaram entre $0,49 \mathrm{e}$ 0,65 , com média de 0,59 .

Resultados comparáveis aos do presente estudo foram observados na avaliação da validade relativa de QFA aplicado com suporte de fotografias com 97 itens em estudo que envolveu 89 adolescentes de Porto Rico, tendo como método de referencia a média de três registros alimentares. A média dos coeficientes de correlação foi de 0,34 e da concordância exata na classificação cruzada em quartis foi de $32,3 \%{ }^{33}$.

No presente estudo, foi observado que o procedimento de correção/deatenuação pela variabilidade intraindividual das estimativas obtidas pelo R24h reduziu os valores superiores dos limites de concordância (LOA) entre o QFA e o R24h, evidenciando que essa correção deve ser empregada por questões metodológicas na estimativa do consumo usual, além de favorecer a comparação entre os instrumentos investigados.

Como limitações deste estudo, podem ser apontados aspectos relativos ao método de referencia e à amostra estudada. Molag et al. ${ }^{34}$, em estudo de meta-análise com 40 artigos de validação de QFA, verificaram que os coeficientes de correlação eram mais elevados quando o método de referência foi aplicado por 8 a 14 dias em comparação a replicações que variavam de um a sete dias. Mais ainda, Pereira et al. ${ }^{35}$ relataram que para adolescentes do Rio de Janeiro a estimativa de consumo usual com erro máximo de $10 \%$ pode requerer até 15 replicações de recordatórios de 24 horas ou registros alimentares. Embora tenham sido empregados procedimentos para minimizar a subestimação no método de referencia, como o uso de imagens de utensílios e a sondagem para alimentos usualmente omitidos, é possível que tenha ocorrido algum grau de subestimação do consumo com o R24h, o que incorreria na minimização dos estimadores da validade do QFA com suporte de fotografias.

Cade et al. ${ }^{2}$ sugerem que participantes de estudos de validação de QFA sejam selecionados 
aleatoriamente na população para a qual o instrumento se destina. Neste estudo, examinou-se amostra selecionada por conveniência, o que é procedimento comum em estudos de avaliação da validade de $\mathrm{QFA}^{17,18}$ e de outras medidas em saúde, como a avaliação da atividade física ${ }^{36-38}$.

Nos últimos anos, o QFA vem sendo considerado um método que incorpora erros na estimativa do consumo ${ }^{39}$, principalmente pela superestimativa, o que também foi verificado no presente estudo, enquanto que o R24h tem sido considerado um método mais acurado para estimar o consumo usual, principalmente se aplicado de forma informatizada pelo menos duas vezes, pois permite a correção para a variabilidade intraindividual $^{40}$. No entanto, estimativas de consumo dietético obtidas com R24h estão sujeitas a erros relacionados aos alimentos consumidos episodicamente e propostas recentes têm recomendado a combinação de QFA e R24h no aprimoramento da estimativa dietética ${ }^{41}$.

O QFA, usualmente, tem sido o método de escolha em estudos de caso-controle e de coorte uma vez que reúne como vantagens a estimativa do consumo usual, que não está sujeita à variabilidade intraindividual e o fato de possibilitar a categorização dos indivíduos em gradientes de consumo, o que permite estimar medidas de associação entre fatores da dieta e diferentes desfechos de saúde e doença. Além disso, estudo de revisão recente de QFA elaborado no Brasil apontou que o número de QFA desenvolvidos no país é insuficiente, considerando o fato de que o país tem dimensões continentais e marcantes diferenças nos hábitos alimentares das diferentes regiões ${ }^{42}$. Assim, este estudo se reveste de importância, dado que não existe outro QFA para adolescentes do Rio de Janeiro.

Cade et al. ${ }^{2}$ indicam que em estudos de validação de QFA, coeficientes de correlação superiores a 0,4 possibilitam a detecção de associações entre a exposição a fatores da dieta e o risco de ocorrência de determinado desfecho. Além disso, verificou-se concordância moderada com base na classificação em quartis para a maioria dos nutrientes analisados, o que indica que o QFA com suporte de fotografias é adequado para categorizar adolescentes do Rio de Janeiro segundo faixas da ingestão usual de energia e nutrientes. Contudo, é importante salientar que as estimativas de ingestão diária de nutrientes obtidas pelo QFA foram superestimadas em média em 30\%.

\section{Conclusão}

O estudo da validade do QFA com suporte de fotografias desenvolvido para adolescentes da região metropolitana do Rio de Janeiro apresentou resultados comparáveis a estudos similares com adolescentes no Brasil e em outros países. Além disso, o suporte de imagens na aplicação do QFA proporcionou resultados mais favoráveis do que os observados na avaliação da validade do QFA original. O QFA com suporte de fotografias de alimentos desenhado para investigar o consumo alimentar de adolescentes apresentou validade relativa aceitável, particularmente para a categorização dos adolescentes segundo gradientes de consumo dietético, constituindo um instrumento útil a ser empregado em estudos sobre consumo alimentar de adolescentes da região metropolitana do Rio de Janeiro.

\section{Colaboradores}

AP Brito colaborou no desenvolvimento do álbum de fotografias de alimentos, concepção do estudo, treinamento e supervisão da equipe de coleta de dados, armazenamento e tratamento dos dados, análise e interpretação dos dados, concepção e redação do manuscrito; MC Araújo na elaboração das fotografias de alimentos, análise e interpretação dos dados, concepção do manuscrito e revisão final; CP Guimarães na elaboração das fotografias de alimentos, concepção do estudo, interpretação dos resultados; RA Pereira na obtenção de recursos, concepção do projeto, coordenação da coleta de dados, análise e interpretação dos dados, concepção do manuscrito e revisão final. 
3. Boeing H. Nutritional epidemiology: New perspectives for understanding the diet-disease relationship? Eur J Clin Nutr 2013; 67(5):424-429.

4. Huybregts L, Roberfroid D, Lachat C, Van Camp J, Kolsteren P. Validity of photographs for food portion estimation in a rural West African setting. Public Health Nutr 2008; 11(6):581-587.

5. Zabotto C, Vianna R, Gil M. Registro fotográfico para inquéritos dietéticos: utensílios e porções. Campinas: $\mathrm{Ne}$ pa-Unicamp; 1996.

6. Lopez R, Botelho R. Identificação de porções alimentares em fotografias. Rev Soc Bras Alim Nutr 2010; 35(1):1-14.

7. Monteiro J, Pfrimer K, Molina M, Tremeschin M, Chiarello P. Consumo alimentar: visualizando porções. São Paulo: Editora Guanabara Koogan; 2006.

8. Araújo M, Santos M. Método visual de alimentos como recurso para inquérito dietético. Salvador: UFBA; 1991.

9. Gonçalves Ferreira M, Pereira RA, Silva RMVG, Sichieri R. Álbum fotográfico para uso em inquéritos alimentares ênfase para a dieta da população mato-grossense. Goiânia: Îndice Gestão Editorial; 2011.

10. Matos SM, Prado MS, Santos CA, D’Innocenzo S, Assis AM, Dourado LS, Oliveira NS, Rodrigues LC, Barreto ML. Validation of a food frequency questionnaire for children and adolescents aged 4 to 11 years living in Salvador, Bahia. Nutr Hosp 2012; 27(4):1114-1119.

11. Zanolla AF, Olinto MT, Henn RL, Wahrlich V, Anjos LA. Assessment of reproducibility and validity of a food frequency questionnaire in a sample of adults living in Porto Alegre, Rio Grande do Sul State, Brazil. Cad Saude Publica 2009; 25(4):840-848.

12. Henn RL, Fuchs SC, Moreira LB, Fuchs FD. Development and validation of a food frequency questionnaire (FFQ-Porto Alegre) for adolescent, adult and elderly populations from Southern Brazil. Cad Saude Publica 2010; 26(11):2068-2079.

13. Crispim SP, Ribeiro RCL, Panato E, Silva MMS, Rosado LEFP, Rosado GP. Validade relativa de um questionário de freqüência alimentar para utilização em adultos. Rev Nutr 2009; 22(1):81-95.

14. Martinez MF, Philippi ST, Estima C, Leal G. Validity and reproducibility of a food frequency questionnaire to assess food group intake in adolescents. Cad Saude Publica 2013; 29(9):1795-1804.

15. Marchioni DM, Voci SM, Lima FE, Fisberg RM, Slater B. Reproducibility of a food frequency questionnaire for adolescents. Cad Saude Publica 2007; 23(9):21872196.

16. Slater B, Enes CC, López RV, Damasceno NR, Voci SM. Validation of a food frequency questionnaire to assess the consumption of carotenoids, fruits and vegetables among adolescents: the method of triads. Cad Saude Publica 2010; 26(11):2090-2100.

17. Slater B, Philippi ST, Fisberg RM, Latorre MR. Validation of a semi-quantitative adolescent food frequency questionnaire applied at a public school in São Paulo, Brazil. Eur J Clin Nutr 2003; 57(5):629-635.

18. Voci SM, Enes CC, Slater B. Validação do Questionário de Freqüência Alimentar para Adolescentes (QFAA) por grupos de alimentos em uma população de escolares. Rev Bras Epidemiol 2008; 11(4):561-572.

19. Araujo MC, Veiga GV, Sichieri R, Pereira RA. Development of a semiquantitative food frequency questionnaire for adolescents from the Rio de Janeiro metropolitan area, Brazil. Rev Nutr 2010; 23(2):179-189.

20. Araujo MC, Ferreira DM, Pereira RA. Reliability of a semi-quantitative food frequency questionnaire designed for adolescents from the Rio de Janeiro Metropolitan Area, Brazil. Cad Saude Publica 2008; 24(12):2775-2786.

21. Araujo MC, Yokoo EM, Pereira RA. Validation and Calibration of a Semiquantitative Food Frequency Questionnaire Designed for Adolescents. J Am Diet Assoc 2010; 110(8):1170-1177.

22. Nelson M, Haraldsdóttir J. Food photographs: practical guidelines II. Development and use of photographic atlases for assessing food portion size. Public Health Nutr 1998; 1(4):231-237.

23. Reichenheim ME, Moraes CL. Alguns pilares para a apreciação da validade de estudos epidemiológicos. Rev Bras Epidemiol 1998; 1(2):132-148.

24. Brito AP, Guimarães CP, Pereira RA. Evaluation of photographs supporting an FFQ developed for adolescents. Public Health Nutr 2012; 17(1):139-144.

25. Conway JM, Ingwersen LA, Vinyard BT, Moshfegh AJ. Effectiveness of the US Department of Agriculture 5-step multiple-pass method in assessing food intake in obese and nonobese women. Am J Clin Nutr 2003; 77(5):1171-1178.

26. Nusser SM, Carriquiry AL, Dodd KW, Fuller WA. A semi parametric transformation approach to estimating usual intake distributions. J Am Stat Assoc 1996; 91:1440-1449.

27. Bland JM, Altman DG. Statistical methods for assessing agreement between two methods of clinical measurement. Lancet 1986; 1(8476):307-310.

28. Ambrosini GL, van Roosbroeck SA, Mackerras D, Fritschi L, de Klerk NH, Musk AW. The reliability of tenyear dietary recall: implications for cancer research. $J$ Nutr 2003; 133(8):2663-2668.

29. Landis JR, Koch GG. The measurement of observer agreement for categorical data. Biometrics 1977; 33(1):159-174.

30. Nurul-Fadhilah A, Teo PS, Foo LH. Validity and reproducibility of a food frequency questionnaire (FFQ) for dietary assessment in Malay adolescents in Malaysia. Asia Pac J Clin Nutr 2012; 21(1):97-103.

31. Kobayashi $T$, Kamimura M, Imai S, Toji C, Okamoto $\mathrm{N}$, Fukui M, Date C. Reproducibility and validity of the food frequency questionnaire for estimating habitual dietary intake in children and adolescents. Nutr J 2011; 24(10):27. 
32. Xia W, Sun C, Zhang L, Zhang X, Wang J, Wang H, Hu L. Reproducibility and relative validity of a food frequency questionnaire developed for female adolescents in Suihua, North China. PLoS One 2011; 6(5):e19656.

33. Preston AM, Palacios C, Rodríguez CA, Vélez-Rodríguez RM. Validation and reproducibility of a semi-quantitative food frequency questionnaire for use in Puerto Rican children. P R Health Sci J 2011; 30(2):58-64.

34. Molag ML, Vries JHM de, Ocké MC, Dagnelie PC, Brandt PA van den, Jansen MCJF, Staveren WA van, Veer P van't. Design characteristics of food frequency questionnaires in relation to their validity. Am J Epidemiol 2007; 166(12):1468-1478.

35. Pereira RA, Araujo MC, Lopes TS, Yokoo EM. How many 24-hour recalls or food records are required to estimate usual energy and nutrient intake? Cad Saude Publica 2010; 26(11):2101-2111.

36. Guedes DP, Lopes CC, Guedes JERP, Stanganelli LC. Reprodutibilidade e validade do questionário Baecke para avaliação da atividade física habitual em adolescentes. Rev Port Cien Desp 2006; 6(3):265-274.

37. Romero A, Florindo AA, Voci SM, Slater B. Reprodutibilidade de questionário informatizado de atividade física em adolescentes. Rev Bras Ativ Fis Saúde 2011; 16(3):234-239.

38. Cabral LGA, Costa FF, Liparotti JR. Evidências preliminares de validade da seção de atividade física do Questionário de Atividade Física e Alimentação do Dia Anterior (QUAFDA). Rev Bras Ativ Fis Saúde 2011; 16(2):100-106.

39. Kipnis V, Subar AF, Midthune D, Freedman LS, Ballard-Barbash R, Troiano RP, Bingham S, Schoeller DA, Schatzkin A, Carroll RJ. Structure of dietary measurement error: results of the OPEN biomarker study. Am J Epidemiol 2003; 158(1):14-21.

40. De Keyzer W, Huybrechts I, De Vriendt V, Vandevijvere S, Slimani N, Van Oyen H, De Henauw S. Repeated 24hour recalls versus dietary records for estimating nutrient intakes in a national food consumption survey. Food Nutr Res 2011; 55.

41. Tooze JA, Midthune D, Dodd KW, Freedman LS, Krebs-Smith SM, Subar AF, Guenther PM, Carroll RJ, Kipnis V. A new statistical method for estimating the usual intake of episodically consumed foods with application to their distribution. J Am Diet Assoc 2006; 106(10):1575-1587.

42. Silva TA, Vasconcelos SML. Procedimentos metodológicos empregados em questionários de frequência alimentar elaborados no Brasil: uma revisão sistemática. Rev Nutr 2012; 25(6):785-797.

Artigo apresentado em 25/05/2015

Aprovado em 14/03/2016

Versão final apresentada em 16/03/2016 